\title{
Non-small cell lung cancer with concomitant intramuscular myxoma of the right psoas mimicking intramuscular metastasis: A case report and literature review
}

\author{
PENG ZHANG $^{1 *}$, XUE MENG $^{1 *}$, LIANKE XIA $^{2}$, PENG XIE $^{1}$, XINDONG SUN $^{1}$, YONGSHENG GAO $^{3}$, \\ SHIJIANG WANG ${ }^{1}$, XIANGUANG ZHAO ${ }^{1}$ and JINMING YU ${ }^{1}$ \\ ${ }^{1}$ Department of Radiation Oncology, Shandong Cancer Hospital and Institute, Jinan; ${ }^{2}$ Department of Orthopedics, \\ Shouguang City People's Hospital, Weifang; ${ }^{3}$ Department of Pathology, Shandong Cancer Hospital and Institute, \\ Jinan, Shandong, P.R. China
}

Received October 31, 2014; Accepted August 17, 2015

DOI: $10.3892 / \mathrm{ol} .2015 .3704$

\begin{abstract}
Intramuscular myxoma (IMM) as a rare soft-tissue tumor arising from the muscles is completely benign. When IMM accompanies malignance, it may be misdiagnosed as muscle metastasis, and for this extremely rare concurrence, the subsequent treatment would vary accordingly. The current study presents, to the best of our knowledge, the first case of non-small cell lung cancer (NSCLC) concomitant with IMM mimicking skeletal muscle metastasis. A 64-year-old female was hospitalized with a history of chest discomfort and right lumbar pain that had persisted for four months. The computed tomography scan showed a lesion in the left upper lobe of the lung and the right psoas, respectively. Serum biomarkers for NSCLC were abnormal. A presumptive clinical diagnosis was compatible with left NSCLC and right psoas muscle metastasis (cT2aN3M1b, stage IV). Stage IV lung cancer would receive palliative treatment. However, the final diagnosis of synchronous left lung squamous cell carcinoma (cT2aN3M0, stage IIIB) and IMM in the right psoas was confirmed by biopsy. The patient therefore underwent definitive chemoradiotherapy for lung carcinoma, and conservative treatment, including analgesics, for IMM. The diagnosis process for a malignant neoplasm concomitant with IMM is not straightforward due to a lack of clinical experience, and it significantly affects the tumor staging and subsequent treatment strategy. The present case suggests that IMM should be included in the differential diagnosis when an abnormal intramuscular lesion concomitant with
\end{abstract}

Correspondence to: Dr Jinming Yu, Department of Radiation Oncology, Shandong Cancer Hospital and Institute, 440 Jiyan Road, Jinan, Shandong 250117, P.R. China

E-mail: sdyujinming@126.com

*Contributed equally

Key words: intramuscular myxoma, intramuscular metastasis, non-small cell lung cancer, psoas malignancy is identified. The value of histopathological diagnosis prior to definitive treatment also requires highlighting.

\section{Introduction}

Myxoma is a true benign neoplasm of mesenchymal origin that produces mucopolysaccharide and immature collagen. Although it is more commonly found in the left atrium of the heart, a myxoma can arise in any region of the body, such as the genitourinary tract, bones, retroperitoneum, skin, joints and skeletal muscles $(1,2)$. When arising from muscle, the tumor is termed an intramuscular myxoma (IMM) (2). IMM is a rare lesion with incidence rates varying between 0.1 and 0.13 per 100,000 individuals per year (3). The benign lesion favors a female predilection, occurring between the fourth and sixth decades of life (4).Wide surgical resection is the most successful form of management, and the prognosis is good (5). Lung cancer is the leading cause of cancer mortality in the United States, with an estimated 224,210 novel cases and 159,260 mortalities in 2014 (6). The malignant tumor can metastasize to almost every region of the body, including skeletal muscle, although this is rather rare (7). There is no consensus on the optimal therapeutic strategy for muscle metastasis, as it may be the end-stage presentation of lung cancer (8). IMM has certain characteristic features, however, when concomitant with a malignant neoplasm, particularly those which possess a high incidence of distant metastases, it may be misdiagnosed as muscle metastasis due to its rare occurrence and particularly rare concurrence $(9,10)$. The current study presents, to the best of our knowledge, the first case of non-small cell lung cancer (NSCLC) concomitant with IMM arising in the right psoas mimicking intramuscular metastasis and briefly reviews the literature concerning this subject.

\section{Case report}

A 64-year-old, non-smoking, female was referred to Shandong Cancer Hospital and Institute (Shandong, Jinan, China) on May 2013 due to a progressive cough with little sputum, 
dyspnea, left chest pain and right lumbar pain that had persisted for four months. The lumbar pain was aggravated upon walking and was relieved spontaneously upon resting. A physical examination revealed a few moist rales, decreased breath sounds over the left lung, and pressing and percussive pain in the right lumbar region. The patient experienced no numbness or tingling in the legs. Examination of the lumbar spine showed normal lumbar lordosis. Sensation in the lower extremity was intact. There was no muscular contracture or palpable mass. Reflexes in the patella and ankle were all symmetrical and normal.

A series of further medical examinations were required for accurate diagnosis. Written informed consent was obtained from the family of the patient. No abnormality was shown on routine laboratory investigations. An initial chest X-ray revealed left lung opacity. Further examinations were also conducted. The serum cytokeratin-19 fragment 21-1 (Cyfra21-1) level was elevated to $7.59 \mathrm{ng} / \mathrm{ml}$ (normal range, 0-3.3 ng/ml). A computed tomography (CT) scan of the chest and abdomen revealed a heterogeneously enhanced $4 \times 5-\mathrm{cm}$ soft-tissue mass with a lobular and spiculate boundary in the left upper lobe, numerous enlarged lymph nodes in the left hilar region and aortopulmonary window. A low-density, well-circumscribed and homogeneous $2.0 \times 2.8-\mathrm{cm}$ mass with mild enhancement was also observed inside the right psoas (Fig. 1A and B). No evidence of distant metastasis was found upon brain magnetic resonance imaging (MRI) or skeletal scintigraphy. Based on the aforementioned examination results, a presumptive clinical diagnosis was compatible with left NSCLC and right psoas muscle metastasis (cT2aN3M1b, stage IV) according to American Joint Committee on Cancer (AJCC) 7th edition staging system for lung cancer (11). The subsequent treatment would be mainly palliative for the end-stage cancer. However, the diagnosis of muscle metastasis was uncertain when considering that the low-density mass with slight enhancement shown by CT did not correspond with the rich vascularization and rapid metabolism of a metastatic lesions, and the fact that there were no abnormalities in the organs susceptible to metastasis, such as the liver and adrenal glands.

To ensure the correct diagnosis for the two lesions, a bronchoscopy and CT-guided puncture biopsy was conducted sequentially. The histology of the lung specimen showed characteristic features of squamous cell lung carcinoma, including mitosis, nuclear hyperchromatism, keratin pearls and intercellular bridges, as expected. However, microscopic analysis of the right psoas muscle specimen showed delicate, monotonous spindle-cells loosely arranged in an abundant myxoid matrix, without evidence of mitotic activity, hemorrhage or necrosis, consistent with IMM (Fig. 2A and B). Thus, the definitive diagnosis was left lung squamous cell carcinoma (cT2aN3M0, stage IIIB) with concomitant IMM arising in the right psoas (11).

For the IMM, although a wide local resection is the most effective treatment and the prognosis for this treatment is excellent; since the patient was elderly, the lesion was benign and there was the presence of highly malignant comorbidity, conservative treatment, consisting analgesics for oral use with regular follow-up was recommended. Concurrent chemoradiotherapy (CRT) is recommended as the best treatment strategy for NSCLC in National Comprehensive Cancer Network guidelines (12), however, it may not have been well tolerated by the patient when considering the poor performance status (Karnofsky performance status score, 70). In fact, the patient was administered sequential CRT for the primary lung cancer consisting of $1,250 \mathrm{mg} / \mathrm{m}^{2}$ gemcitabine on days 1 and 8 , plus $75 \mathrm{mg} / \mathrm{m}^{2}$ cisplatin on day 1 , every 21 days for 4 cycles, followed by definitive 3 -dimensional conformal radiotherapy with a total dose of $60 \mathrm{~Gy}$ in $2 \mathrm{~Gy}$ daily fractions. The primary lung cancer lesion and metastatic lymph nodes were reduced slightly, and the symptoms associated with lung cancer were relieved after 2 cycles of chemotherapy and further improved once the chemotherapy plan was completed. The efficacy of treatment was assessed as partial remission. Subsequent to half a year of follow-up, the symptoms of coughing, dyspnea and left chest pain were completely resolved. The right lumbar pain still existed, but was relieved by analgesics. The serum Cyfra21-1 level was decreased to normal at $1.15 \mathrm{ng} / \mathrm{ml}$. The CT reexamination demonstrated that the lesion size in the left lung had significantly decreased to $1.5 \times 2.0 \mathrm{~cm}$ compared with pre-treatment and that there was no marked change of the IMM (Fig. 1C and D). The patient received supportive care and was followed up every 3 months. Ten months later, a skeletal scintigraphy revealed the presence of bone metastases. One month later, 17 months after diagnosis, the patient succumbed to the disease.

\section{Discussion}

In the present case, the chest discomfort and lumbar pain appeared almost at the same time. The abnormal lesions in the left lung and right psoas were detected by CT simultaneously. The CT manifestation of the lung lesion and the serum specific biomarker of the tumor supported the diagnosis of NSCLC. However, the nature of the psoas lesion could not be ascertained. Although the CT presentation did not conform to the typical skeletal muscle metastasis, which is rather rare, this suspicious diagnosis could not be ruled out solely relying on the imaging findings, particularly considering that NSCLC is prone to distant metastases (7). The differential diagnosis should include intramuscular metastasis and primary soft-tissue tumors, as each can arise from the psoas muscle and cause lumbar pain (13-15). Furthermore, the definition of the nature of the muscle abnormality would be of great value, as the correct diagnosis would affect the following clinical management and vary the prognosis of the patient. The aspects mentioned next may be beneficial in making a differential diagnosis.

With regard to the epidemiology, a large portion of patients suffering from NSCLC are diagnosed at an advanced stage with distant metastases. The most commonly involved sites are the liver (33-40\%), abdominal lymph nodes (29\%), adrenal glands (18-38\%), bones (19-33\%), brain (15-43\%) and kidneys (16-23\%) (16). In general, hematogenous skeletal muscle metastasis is rare and the metastasis of lung cancer is even rarer, although it is the most common source (17). The accurate incidence of skeletal muscle metastasis is uncertain, although has been reported as $0.8 \%$ in patients with cancer and $1 \%$ in patients with lung cancer through autopsy findings. Psoas metastases are extremely rare, with only few cases having been reported $(17,18)$. The trunk musculature (particularly the 


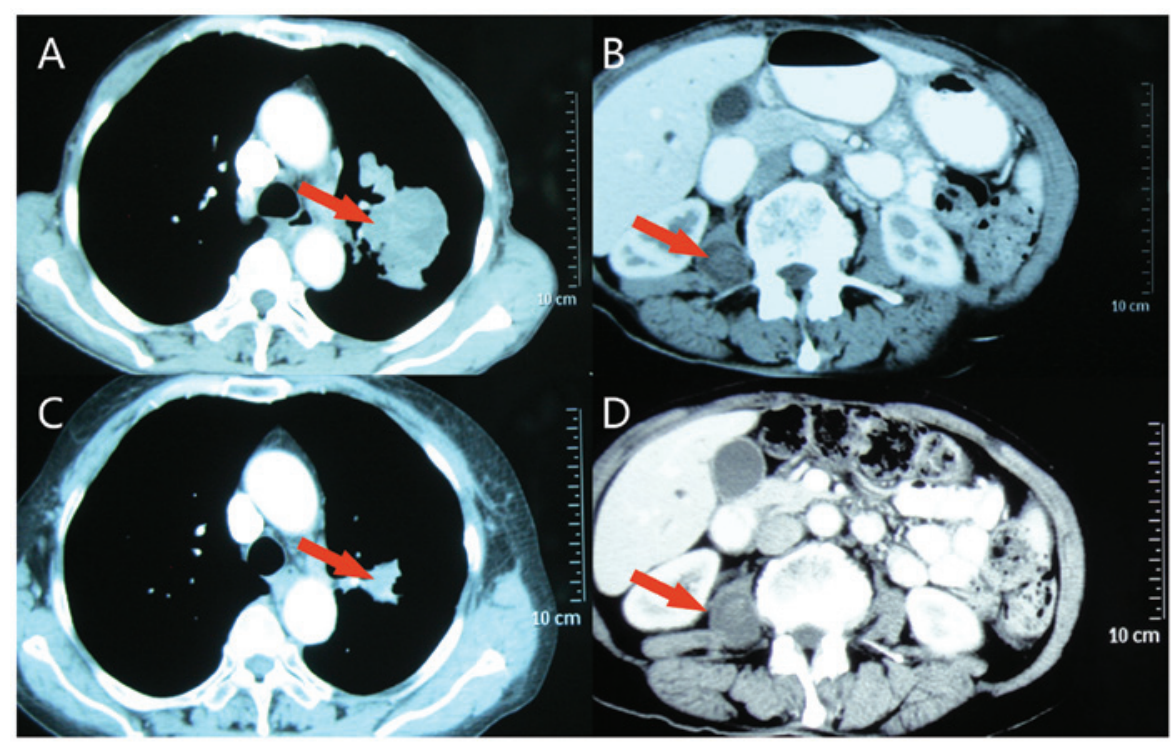

Figure 1. (A) Chest CT showing a heterogeneously enhanced 4x5-cm soft-tissue mass with lobular and spiculate boundaries in left upper lobe and numerous enlarged lymph nodes in the left hilar region and aortopulmonary window. (B) Abdominal CT showing a well-circumscribed, $2.0 \mathrm{x} 2.8 \mathrm{~cm}$, homogeneous mass inside the right psoas muscle, with low-density mild enhancement. CT after 6 months showing that (C) the lesion size in the left lung had significantly decreased to $1.5 \times 2.0 \mathrm{~cm}$ and that (D) there was almost no change in the intramuscular myxoma compared with pre-treatment. CT, computed tomography.

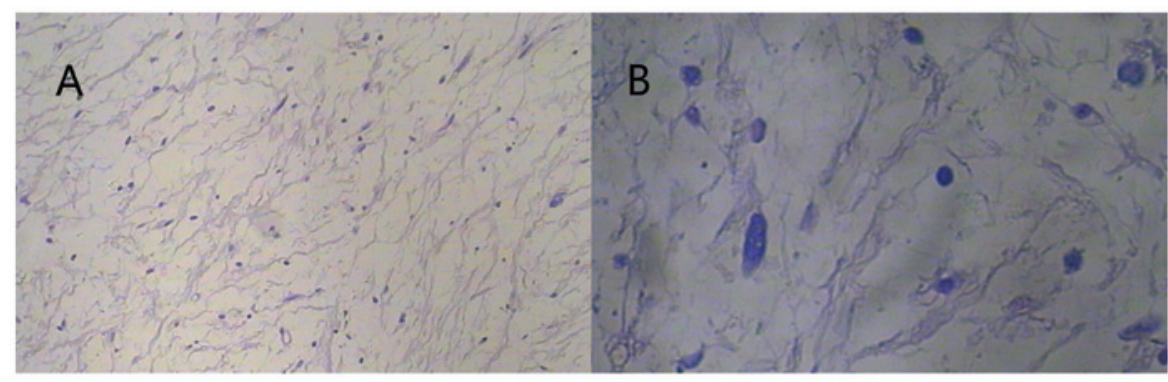

Figure 2. Hematoxylin and eosin stain at (A) x100 magnification showing a hypocellular and hypovascular lesion rich in abundant myxoid matrix, with undifferentiated bland stellate or spindle-shaped cells separated by reticulin fibers. (B) Higher-power magnification (x400) demonstrating the charcteristic stellate or spindle pattern of cells loosely arranged in an abundant myxoid matrix.

paravertebral and iliopsoas muscles) and the thigh muscles are the most frequent sites (19).

IMM, however, is much more rare (3). IMM generally occur in the large muscles of the thighs, shoulders, buttocks or upper arm, in order of descending frequency (20). These rare lesions mainly occur between the fourth and sixth decades of life, with a slight predominance in women due to unknown reasons (4). As no capsule encompasses them, the lesions may infiltrate the adjacent tissues occasionally without metastasis (21). Only four cases of psoas IMM have been reported in the literature to date (14,22-24), and no case has been recorded to be concomitant with malignancy. So, when this does occur, the abnormality is difficult to diagnose as IMM rather than a metastasis.

The most frequent clinical manifestation of muscle metastasis is local pain, with or without a palpable mass (15). In individual cases, skeletal muscle metastasis from lung cancer has even presented as the initial clinical manifestation, so it may be easily misdiagnosed, particularly when the primary tumor is unknown (25). However, the symptoms of IMM depend largely on the location and size of the lesion, with presentation as a solitary slow-growing painless mass, or secondary to compression of adjacent structures, mainly nerves and vessels, although $\sim 80 \%$ of cases are asymptomatic. Two of the reported cases in the literature complained of pain in the region associated with the psoas (14).

IMM and intramuscular metastasis can each arise from the psoas muscle and cause lumbar pain, as reported in the literature. Therefore, it is not possible to distinguish between them based on the presentation only. Their imaging characteristics are also required for the differential diagnosis. As the tumors are rare in the clinic, experience to diagnose them is clearly lacking. Relevant knowledge is mostly obtained from previous case studies. Intramuscular metastasis usually manifests as a rim-enhancing focus with a central hypoattenuation likely due to necrosis (26). CT findings of IMM, as shown in Fig. 1, are a cystic, well-circumscribed, low-attenuated and homogeneous mass within the skeletal muscle (20). The mass exhibits a high content of mesenchyme, with few vascular and no internal calcifications, and as a result will show mild or no enhancement following intravenous administration of contrast medium. The two lesions show similar MRI features of a high signal intensity on T2-weighted images, and a low or isointense signal intensity on T1-weighted images $(23,27)$. 
Published studies on the use of CT or MRI suggest that an imaging examination cannot accurately determine the psoas muscle pathology (28), and the diagnosis of these lesions is not usually straightforward, even with radiological imaging. Muscle metastasis can, for example, mimic a benign lesion such as an abscess, as previously reported (29). As a result, the value of histopathological evaluation is highlighted in the differential diagnosis.

For histopathological evaluation, image-guided biopsy and fine-needle aspiration cytology are frequently required to confirm the nature of the lesions. For IMM, microscopic examination shows a hypocellular and hypovascular tumor with undifferentiated bland stellate or spindle-shaped cells, separated by reticulin fibers in abundant myxoid matrix of hyaluronic acid sparse. It shows the benign characteristics with the absence of mitosis, nuclear atypia and necrosis $(20,24)$. The majority of lesions infiltrate into the adjacent muscles, as the delicate pseudocapsules are incomplete on close inspection (30). On immunohistochemical staining, IMM cells stain positively for vimentin, and variably for CD34 and actin; immunostain for S-100 protein is typically negative, unlike in myxoid liposarcoma and neurothekeoma (nerve sheath myxoma) (30). As the metastatic lesion is transferred hematogenously, it will show malignant characteristics, such as mitosis, nuclear atypia, necrosis and specific immunohistochemical results corresponding with the primary tumor site (31). In the present study, a rather rare lesion, namely an IMM, was unexpectedly found in the psoas, with left lung squamous cell carcinoma.

If a diagnosis of muscle metastasis is ascertained, it may be the end-stage presentation of lung cancer portending a poor prognosis. There has been no unified optimal strategy put forward for skeletal muscle metastases. Although chemotherapy, radiotherapy and even surgical excision may be applied for local control or palliation, it is unknown whether these therapeutic methods can achieve a survival benefit (32). In fact, the majority of patients with skeletal muscle metastasis from lung cancer succumb within a few months, despite various forms of treatment being administered (33). On the other hand, lung cancer at stage IIIB should receive chemotherapy and definitive radiotherapy. In the present study, the anticancer treatment was not affected by the coexistence of IMM. The dominating treatment for IMM is wide local excision for its absolutely benign nature. A recent retrospective study of IMM over 10 years found no recurrence after resection (34). The prognosis of IMM is excellent if the tumor is completely excised with a pathological safe margin.

In conclusion, this report represents the first case of lung cancer coexisted with IMM, which was initially suspected to be intramuscular metastasis. The two lesions are quite rare in daily clinical work, so there is a lack of experience for forming a differential diagnosis. When a soft-tissue lesion is concomitant with malignant carcinoma, a primary soft-tissue tumor and muscle metastasis should be considered. In the present case, the nature of the lesion in the psoas muscle determined the lung cancer stage and significantly affected the following treatment strategy. Although imaging studies can offer certain characteristic information, histopathological examination remains the gold standard and its importance should be highlighted. This case indicates that extreme caution is essential in forming a diagnosis when a soft-tissue lesion is concomitant with malignancy. IMM should be considered in the differential diagnosis.

\section{Acknowledgements}

The authors would like to acknowledge Shandong Cancer Hospital and Institute for providing the details of this rare case.

\section{References}

1. Stout AP: Myxoma, the tumor of primitive mesenchyme. Ann Surg 127: 706-719, 1948.

2. Allen PW: Myxoma is not a single entity: A review of the concept of myxoma. Ann Diagn Pathol 4: 99-123, 2000.

3. Heymans O, Gebhart M, Alexiou J, de Saint Aubain N and Larsimont D: Intramuscular myxoma. Acta Chir Belg 98: 120-122, 1998.

4. Enzinger FM: Intramuscular myxoma; A review and follow-up study of 34 cases. Am J Clin Pathol 43: 104-113, 1965.

5. Charron P and Smith J: Intramuscular myxomas: A clinicopathologic study with emphasis on surgical management. Am Surg 70:1073-1077, 2004.

6. Siegel R, Ma J, Zou Z and Jemal A: Cancer statistics, 2014. CA Cancer J Clin 64: 9-29, 2014.

7. Kwas HH, Zendah I and Ghedira H: Skeletal muscle metastases from lung cancer. Asian Cardiovasc Thorac Ann 21: 741-743, 2013.

8. Pop D, Nadeemy AS, Venissac N, Guiraudet P, Otto J, Poudenx M and Mouroux J: Skeletal muscle metastasis from non-small cell lung cancer. J Thorac Oncol 4: 1236-1241, 2009.

9. Falavigna A, Righesso O, Volquind D and Teles AR: Intramuscular myxoma of the cervical paraspinal muscle. Eur Spine J 18: 245-249, 2009.

10. Ripalda E, Beni R,Reguero ME, Nistal J and Carda P: Recurrent Intramuscular Psoas Myxoma. Am Surg 75: 862-863, 2009.

11. Goldstraw P, Crowley J, Chansky K, Giroux DJ, Groome PA, Rami-Porta R, Postmus PE, Rusch V and Sobin L; International Association for the Study of Lung Cancer International Staging Committee; Participating Institutions: The IASLC Lung Cancer Staging Project: Proposals for the revision of the TNM stage groupings in the forthcoming (seventh) edition of the TNM Classification of malignant tumours. J Thorac Oncol 2: 706-714, 2007.

12. Aupérin A, Le Péchoux C, Rolland E, Curran WJ, Furuse K, Fournel P, Belderbos J, Clamon G, Ulutin HC and Paulus R: Meta-analysis of concomitant versus sequential radiochemotherapy in locally advanced non-small-cell lung cancer. J Clin Oncol 28: 2181-2190, 2010.

13. Sudo A, Ogihara Y, Shiokawa Y, Fujinami S and Sekiguchi S: Intramuscular metastasis of carcinoma. Clin Orthop Relat Res 296: 213-217, 1993.

14. Ruiz-Tovar J, Ripalda E, Beni R, Reguero ME, Nistal J and Carda P: Recurrent intramuscular psoas myxoma. Am Surg 75: 862-863, 2009.

15. Damron TA and Heiner J: Distant soft tissue metastases: A series of 30 new patients and 91 cases from the literature. Ann Surg Oncol 7: 526-534, 2000.

16. Quint LE, Tummala S, Brisson LJ, Francis IR, Krupnick AS, Kazerooni EA, Iannettoni MD, Whyte RI and Orringer MB: Distribution of distant metastases from newly diagnosed non-small cell lung cancer. Ann Thorac Surg 62: 246-250, 1996.

17. Ampil FL, Lall C and Datta R: Palliative management of metastatic tumors involving the psoas muscle: Case reports and review of the literature. Am J Clin Oncol 24: 313-314, 2001.

18. Strauss JB, Shah AP, Chen SS and Gielda BT, Kim AW: Psoas muscle metastases in non-small cell lung cancer. J Thorac Dis 4: 83-87, 2012.

19. Plaza JA, Perez-Montiel D, Mayerson J, Morrison C and Suster S: Metastases to soft tissue: A review of 118 cases over a 30-year period. Cancer 112: 193-203, 2008.

20. Murphey MD, McRae GA, Fanburg-Smith JC, Temple HT, Levine AM and Aboulafia AJ: Imaging of soft-tissue myxoma with emphasis on CT and MR and comparison of radiologic and pathologic findings. Radiology 225: 215-224, 2002.

21. Bancroft LW, Kransdorf MJ, Menke DM, O'Connor MI and Foster WC: Intramuscular myxoma: Characteristic MR imaging features. AJR Am J Roentgenol 178: 1255-1259, 2002. 
22. Tassart M, Roussy M, Boidart F and Tricot JF: Muscular myxoma. A case of myxoma of the psoas. J Radiol 74: 147-150, 1993.

23. van Roggen JF, McMenamin ME and Fletcher CD: Cellular myxoma of soft tissue: A clinicopathological study of 38 cases confirming indolent clinical behaviour. Histopathology 39: 287-297, 2001.

24. Dormand EL, Prabhu-Desai A, Rice AJ and Rosin RD: Not all pain in the left iliac fossa is diverticular disease: A case study of a psoas myxoma and review. Surgeon 4: 239-243, 2006.

25. Haraguchi N, Yamamoto Y, Sasaki K, Satoh H and Sekizawa K Muscle metastases as initial manifestation of lung cancer. Clin Oncol (R Coll Radiol) 16: 586-587, 2004.

26. Pretorius ES and Fishman EK: HelicalCT of skeletal muscle metastases from primary carcinomas. AJR Am J Roentgenol 174: 401-404, 2000

27. Williams JB, Youngberg RA, Bui-Mansfield LT and Pitcher JD: MR imaging of skeletal muscle metastases. AJR Am J Roentgenol 168: 555-557, 1997.

28. Yang WT, Yeo W and Metreweli C: Imaging of iliopsoas metastasis. Clin Radiol 54: 85-89, 1999.
29. Stewart IC, Blaikie KJ and MacLeod HM: Adenocarcinoma of unknown primary site (ACUPS) presenting as a psoas abscess. Scott Med J 34: 470, 1989.

30. Ozawa H, Fujii M, Tomita T and Ogawa K: Intramuscular myxoma of scalene muscle: A case report. Auris Nasus Larynx 31: 319-322, 2004.

31. Molina-Garrido MJ and Guillén-Ponce C: Muscle metastasis of carcinoma. Clin Transl Oncol 13: 98-101, 2011

32. Al-Alao BS, Westrup J and Shuhaibar MN: Non-small-cell lung cancer: Unusual presentation in the gluteal muscle. Gen Thorac Cardiovasc Surg 59: 382-384, 2011.

33. McKeown PP, Conant P and Auerbach LE: Squamous cell carcinoma of the lung: An unusual metastasis to pectoralis muscle. Ann Thorac Surg 61: 1525-1526, 1996.

34. Charron P and Smith J: Intramuscular myxomas: A clinicopathologic study with emphasis on surgical management. Am Surg 70: 1073-1077, 2004. 